\title{
Characterizing the Geometric Detection Efficiency of EDX Detectors
}

\author{
Frederick H. Schamber
}

Retired: 5095 Cherry Drive, Murrysville, PA 15668, frederick.schamber@gmail.com

Energy Dispersive X-ray analysis (EDX) has been a powerful tool for microanalysis since the early 1970’s. However, as SDD (Silicon Drift Detector) technology has supplanted the older Si(Li) (Lithium drifted Silicon) detector it has created the opportunity for a wide range of detector sizes and configurations that were not previously practical. Whereas sensor area was previously a crude but reasonably reliable means for predicting the relative collection efficiency of EDX detectors, such is no longer the case and sensor area is today a very inadequate predictor of actual performance, as is illustrated in Fig. 1.

Geometric collection efficiency (GCE) is defined as the probability that an x-ray emitted from a given point on the specimen will be incident on the active area of the sensor, subject to geometric considerations only. In other words, this definition is intended to be independent of efficiency factors such as energy-dependent window absorption, sensor stopping power, count rate dependence, analyzer system settings, etc.

The relative solid angle $(\Omega / 4 \pi)$ is, of course, the definitive mathematical expression of GCE and Zaluzec has provided comprehensive aids for performing accurate calculations of solid angle $(\Omega)$ from known detector dimensions [1,2,3]. However, solid angle is not a quantity that can be directly measured, and must be computed from dimensions that are not readily available to an end-user. Further, a true assessment of effective solid angle must necessarily take into account such things as occlusion by the support grid of the detector window and vignetting by the electron trap - factors that become increasingly problematic when the x-rays do not originate on the detector's axis. Thus, a calculation of solid angle, though a useful statement of how a detector should perform under specific idealized conditions, is not necessarily representative of how the detector might actually be performing in a given configuration. And finally, the mathematical abstraction of a solid angle value expressed in steradians does not have obvious intuitive meaning for most individuals.

Microscopists experience the effects of geometric collection efficiency in terms of count rate realized for a given beam current and do quickly develop an intuitive sense for this relationship. Thus the ratio "counts/sec-nA" is a natural empirical metric. Such measurements are extremely easy to make with only minimal equipment and provide a measure of detector performance that naturally extrapolates to other situations. However, without a consistent methodology, such measurements remain subjective assessments that cannot readily be compared between different users and laboratories nor readily related to formal solid angle calculations.

The present proposal is to employ the effective solid angle $\left(\Omega_{\text {eff }}\right)$ as the standard theoretical/mathematical expression of GCE, where $\Omega_{\text {eff }}$ incorporates the effect of all opaque obstructions such as window support grid, specimen holder, and electron-trap collimation effects. Because of the complexity of quantifying such obstructions for off-axis x-rays, $\Omega_{e f f}$ will typically be computed for the ideal case where the beam is incident at a point on the detector axis.

It is then further proposed that a standard empirical metric of GCE be defined as the kilocounts/second/nanoamp for the measured K series x-rays, corrected for loss fraction (i.e. “dead time”), 
on a smooth pure copper specimen excited at normal incidence with a $20 \mathrm{KV}$ electron beam. This metric, expressed as KCPS/nA (CuK @ 20KV) is proposed because: (a) it is easily measured with basic instrumentation; (b) it is intuitively meaningful; (c) it is insensitive to the settings (e.g., time constants or thresholds) of the x-ray analyzer; and (d) it can be directly related to $\Omega_{\text {eff }}$ by the relationship:

$$
\frac{I C R}{I_{\text {beam }}}=C(\theta) \Omega_{\text {eff }}
$$

where $I_{\text {beam }}$ is the beam current incident on the specimen and ICR is the true "input count rate" of CuK events presented to the X-ray analyzer system. (X-ray analyzers conventionally infer ICR by dividing the actual measured counts by an effective "live time".) The constant $C(\theta)$ is the probability that a copper $\mathrm{K} \alpha$ or $\mathrm{K} \beta$ x-ray is emitted with a takeoff angle of $\theta$ when a $20 \mathrm{KV}$ electron is normally incident on a flat copper specimen. A table of $C(\theta)$ values therefore permits easy conversion between the empirical measure of GCE and its formal expression in solid angle.

This proposal is being submitted for consideration by the USA Technical Advisory Group of ISO/TC202 dealing with standard practices for microbeam analysis.

\section{References:}

[1] N.J. Zaluzec, “Calculating the Detector Solid Angle in X-ray Energy Dispersive Spectroscopy”, Microscopy and Microanalysis, 15[Suppl. 2] (2009) 520-521.

[2] N.J. Zaluzec, “Analytical Formulae for Calculation of X-Ray Detector Solid Angles in the Scanning and Scanning Transmission Analytical Electron Microscope”, Microscopy and Microanalysis, 20[4] (2014) 1318-1326.

[3] N.J. Zaluzec, “XEDS Tools: Solid Angle Calculator”, Website: http://tpm.amc.anl.gov/NJZTools/XEDSSolidAngle.html

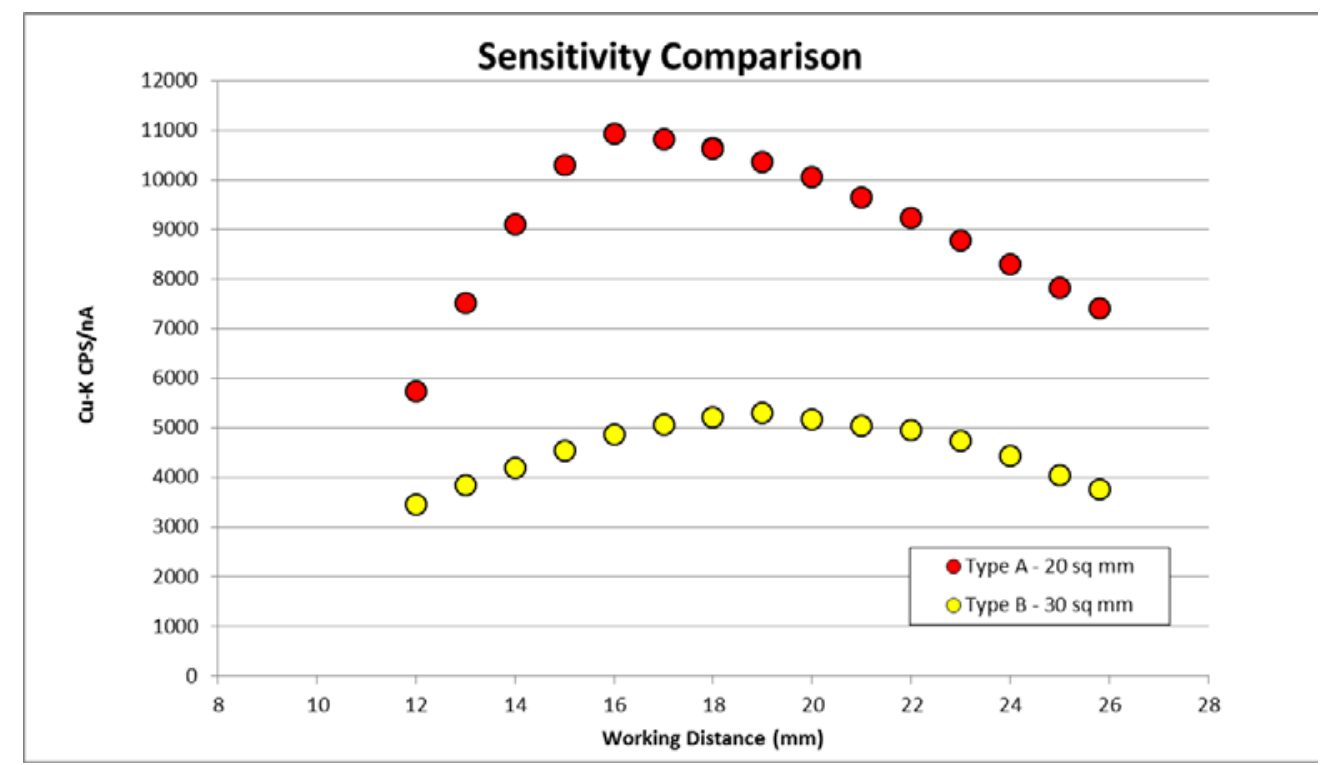

Figure 1 - Sensitivity comparison of two detector types for copper $\mathrm{K}$ x-rays. Type A has twice the peak sensitivity even though the sensor is only $2 / 3$ the area of Type B. (Both $20 \mathrm{KV}$ on pure Cu.) 